\title{
Influence of load increment ratio on one-dimensional consolidation behaviour
}

\author{
Takahiro Yoshidomi ${ }^{1}$, Koichi Iinuma ${ }^{2}$, Motohiro Sugiyama ${ }^{3 *}$ \\ ${ }^{1}$ Course of Architecture and Civil Engineering, School of Engineering, Tokai University, Kanagawa, Japan \\ ${ }^{2}$ Design Department, Tokyo Branch, Ohba Co., Ltd., Tokyo, Japan \\ ${ }^{3}$ Department of Civil Engineering, Faculty of Engineering, Tokai University, Kanagawa, Japan
}

\begin{abstract}
In this study, we conducted a stage loading consolidation test with varying load increment ratio and investigated its effect. By adjusting the primary consolidation ratio of one-dimensional consolidation analysis with secondary consolidation taken into account, it is possible to reproduce the consolidation amount-time curve affected by the load increment ratio.
\end{abstract}

\section{Introduction}

In the incremental loading consolidation test (JIS A 1210), load increment ratio $\Delta p / p$ is set to 1 and loaded at one-day intervals. $\Delta p / p=1$ is considerably excessive as compared with loading and construction conditions at the site. The incremental ratio for load distribution due to local load on thick clay layer and low loading such as low embankment and housing is much smaller than 1. It has been pointed out that the $e-\log p$ curve of the test with varying loading conditions (loading time interval, load increment ratio, and strain rate) varies greatly as shown in Figure 1 (a) and (b)[1-3]. On the other hand, some samples show different results from previous studies[4]. Showing the presence of such a resultant sample leads to settlement prediction accuracy. It is also a well-known fact that when the increment ratio is small, the consolidation-time curve becomes convex upwards and does not become an inverted S-shaped settlement curve[5]. As the secondary compression is dominant, the primary consolidation may be less than half of the total consolidation in some cases.

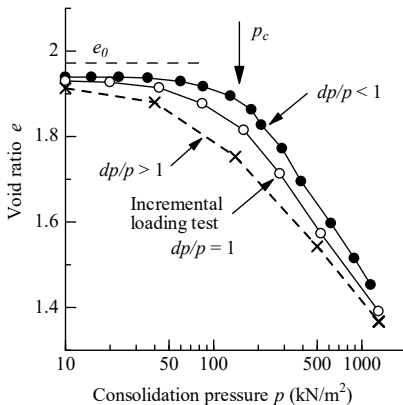

(a) Influence of load incremental ratio

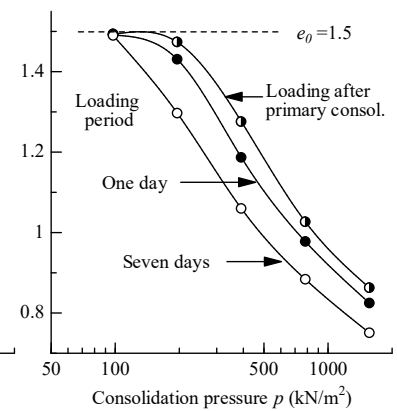

(b) Influence of loading time interval
Figure 1. Influence of loading condition on the $e-\log p$ curve [2]

In this study, an incremental loading consolidation test was performed by varying the load incremental ratio to investigate its effects. It shows that adjusting the primary consolidation ratio of one-dimensional consolidation analysis considering secondary consolidation can reproduce the consolidation-time curve subject to the effect of the load incremental ratio.

\section{Samples and test method}

Four kinds of undisturbed clayey soil (U1 to U4) and two kinds of reconstituted clayey soil (R1, R2) were used as samples. Table 1 shows the physical properties of each sample. Samples U3 and R2 are high organic soils. Loading was carried out from $p=9.81 \mathrm{kN} / \mathrm{m}^{2}$ with a load increment ratio of 0.2 to 1.5 , and the loading time of each load was adjusted so that the consolidation period was all 8 days. In addition, the loading $\Delta p=39.2$ to 235.5 $\mathrm{kN} / \mathrm{m}^{2}$ was loaded on six U4 samples consolidated at $p=157 \mathrm{kN} / \mathrm{m}^{2}$ for 2 days, respectively, and the settlement was measured for 24 hours.

Table 1. Physical properties of clayey soil

\begin{tabular}{|c|c|c|c|c|c|c|}
\hline Sample & U1 & U2 & U3 & U4 & R1 & R2 \\
\hline$\rho_{s}$ & 2.55 & 2.69 & 1.62 & 2.70 & 2.67 & 2.31 \\
\hline $\mathrm{LL}(\%)$ & 64.1 & 67.8 & 525 & 96 & 68.4 & 201.8 \\
\hline $\mathrm{PI}(\%)$ & 29.4 & 33.3 & 370 & 52 & 44.6 & 68.2 \\
\hline Sand(\%) & 5 & 0 & - & 11 & 5 & - \\
\hline Silt(\%) & 50 & 41 & - & 33 & 42 & - \\
\hline Clay(\%) & 45 & 59 & - & 56 & 53 & - \\
\hline
\end{tabular}

\section{Test results and consideration}

\subsection{Influence of load increment ratio}

The relationships of volumetric strain (=axial strain) with $\log p$ of the undisturbed and reconstituted samples

\footnotetext{
* Corresponding author: sugi@keyaki.cc.u-tokai.ac.jp
} 


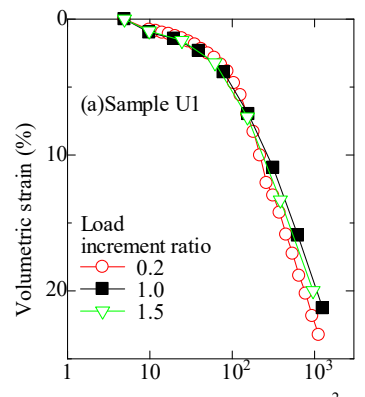

Consolidation pressure $p\left(\mathrm{kN} / \mathrm{m}^{2}\right.$
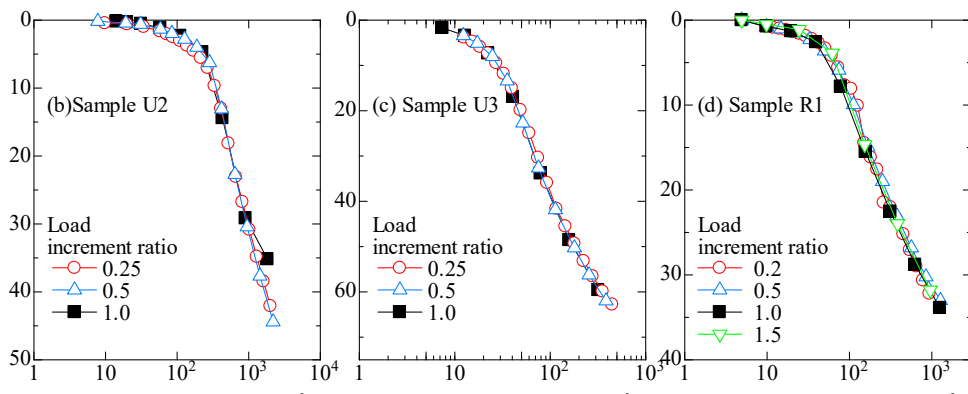

Consolidation pressure $p\left(\mathrm{kN} / \mathrm{m}^{2}\right)$

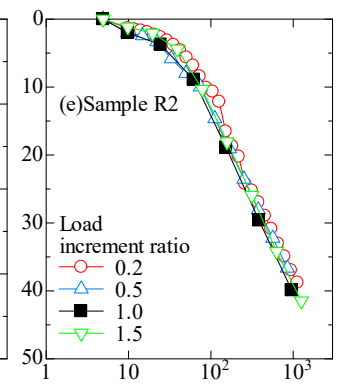

Consolidation pressure $p\left(\mathrm{kN} / \mathrm{m}^{2}\right)$

Figure 2. $\varepsilon-\log p$ curve varying with a load incremental ratio

are shown in Figure 2(a) to (e). In the undisturbed samples, there was scattering of the initial void ratio $e$ in particular, for this study, the volumetric strain was sorted out instead of the void ratio $e$.

Regardless of the sample and load increment ratio, there is no significant difference in each $e-\log p$ relationship as in Figure 2. A similar result was observed in undisturbed undersea clay in Osaka Bay[4], which contrasts with past conspicuous results[1]. In the conceptual diagram relating to delayed compression of Bjerrum, the $e-\log p$ curves with different consolidation times are parallel to each other as shown in Figure 3, the compression index $C c$ is equal, and the parallel lines are assumed to be strain rate constant lines[3]. Therefore, the strain rates in Figure 4 shows the strain rate in Figure 2 where the $\varepsilon-\log p$ curves became almost equal despite the difference in the load increment ratio. Even though the $\varepsilon-$ $\log p$ curves are nearly equal, the strain rate changes in each test. This is contrary to the fact that the strain rate on the $e-\log p$ curve assumed by Bjerrum is equal.

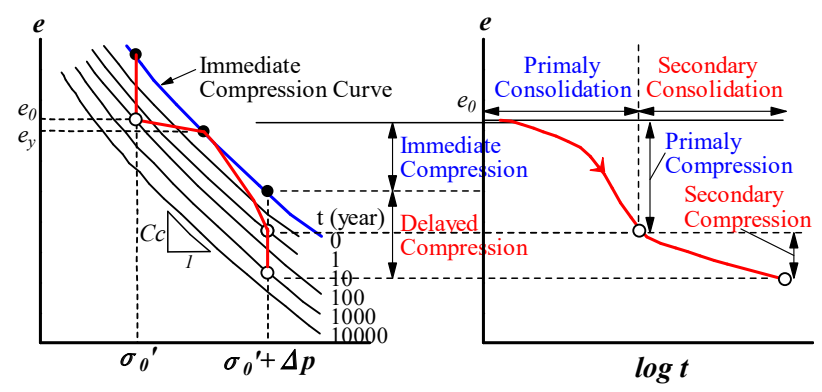

Figure 3. Conceptual figure of Bjerrum's delayed consolidation $^{[3]}$
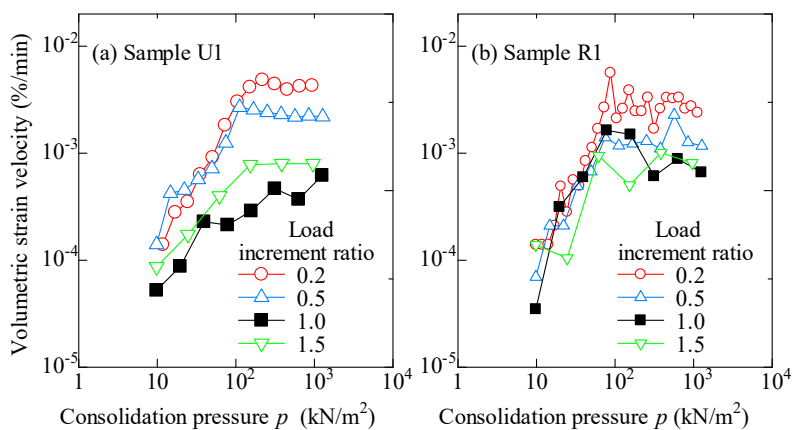

Figure 4. Load incremental ratio and volumetric strain rate

\subsection{Load incremental ratio and consolidation- time curve}

\subsubsection{Change of consolidation-time curve}

The symbol in Figure 5 is the measured value of the consolidation test with changing the load increment ratio. The figure shows that the shape of consolidation-time curve varies with decreasing load increment ratio as in previous studies, and it is not a typical inverted $\mathrm{S}$ shape. Because of the change in the shape of the curve, the end of primary consolidation time is unclear. However, the influence of the load increment ratio on the secondary consolidation is not significant, and the difference in the coefficient of secondary consolidation of each test is small.

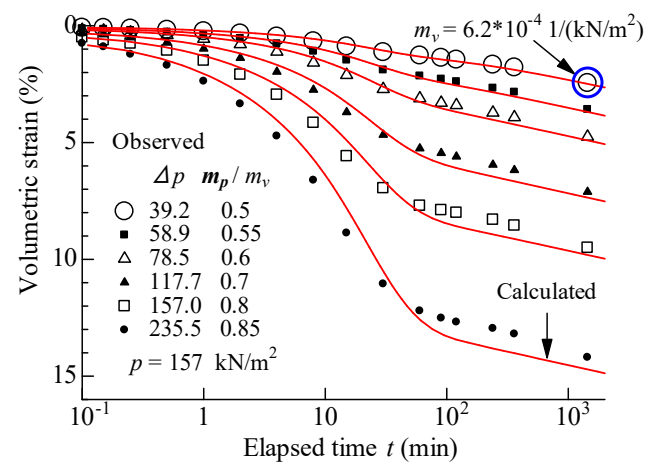

Figure 5. Consolidation-time curve: measured values and calculation results

\subsubsection{One-dimensional consolidation equation and secondary consolidation model[6]}

It is thought that the total volumetric strain rate $\dot{\varepsilon}_{i}\left(=\varepsilon_{f} / t_{f}\right)$ in one-dimensional consolidation is divided into primary and secondary consolidation components.

$$
\dot{\varepsilon}\left(=\frac{\partial \varepsilon}{\partial t}\right)=\dot{\varepsilon}_{p}\left(=-m_{p} \cdot \dot{u}\right)+\dot{\varepsilon}_{S}
$$

where $\dot{\varepsilon}_{p}$ and $\dot{\varepsilon}_{s}$ are the primary and secondary consolidation rates respectively, and $m_{p}$ is the coefficient of volume compressibility defined by primary consolidation (volumetric strain). Substituting Eq. (1) in the continuous condition Eq. (1) that governs onedimensional consolidation gives Eq. (2). 


$$
\frac{\partial u}{\partial t}=c_{v}^{*} \frac{\partial^{2} u}{\partial y^{2}}+\dot{\varepsilon}_{s} / m_{p}
$$

where $c_{v}{ }^{*}\left(k /\left(\gamma_{w}{ }^{*} m_{p}\right)\right)$ is the coefficient of consolidation that is defined using the primary consolidation, and the second term on the right side $\dot{\varepsilon}_{s} / m_{p}$ is the excess pore water pressure created by secondary consolidation. $\dot{\varepsilon}_{s}$ is obtained by Eq. (3) according to the secondary consolidation $\Delta \varepsilon_{s}$, the coefficient of secondary consolidation $\alpha$ defined by the volumetric strain and the initial rate of secondary consolidation $\dot{\varepsilon}_{i}$.

$$
\dot{\varepsilon}_{s}=\dot{\varepsilon}_{i} \cdot \exp \left(\frac{-\Delta \varepsilon_{s}}{\alpha}\right)
$$

Since the secondary consolidation behavior during primary consolidation is unknown and $\dot{\varepsilon}_{i}$ can not be experimentally investigated, $\dot{\varepsilon}_{i}$ is assumed in onedimensional consolidation analysis. The authors propose to calculate the initial rate of the secondary consolidation from Eq.(4) using the secondary consolidation $\Delta \varepsilon_{s f}$ and its rate $\dot{\varepsilon}_{s f}=\alpha / 1(1 /$ day $)$ after one day of the incremental loading consolidation test [6].

$$
\dot{\varepsilon}_{i}=\dot{\varepsilon}_{s f} \cdot \exp \left(\frac{\Delta \varepsilon_{s f}}{\alpha}\right)
$$

With this proposed method, as the constant that represents the primary consolidation, the coefficient of volumetric consolidation $m_{p}$ is defined and distinguished from ordinary $m_{v}$. Since $m_{p}$ cannot be determined experimentally, so it need to assume. The consolidationtime curve is calculated based on the one-dimensional consolidation equation, Eq. (2), considering secondary consolidation, and by the explicit differential method according to the following boundary conditions and initial conditions.

$$
\begin{array}{lll}
t=0 \quad \& \quad H \geq y \geq 0 ; & u=u_{0}\left(=\Delta \sigma_{y}\right) \\
t \geq 0 \quad \& \quad y=H ; & \partial u / \partial y=0 \\
t>0 \quad \& y=0 ; & u=0
\end{array}
$$

where $H$ is the maximum drainage distance and $u_{0}$ is the initial excess pore water pressure. The soil constants needed for the calculation are $m_{v}, c_{v}, \alpha$, and $m_{p}$. With the proposed method, $\dot{\varepsilon}_{i}$ is calculated by assuming $m_{p}$ or the primary consolidation ratio.

The solid line in Figure 5 shows the results based on the consolidation-time curve of $\Delta p=39.2 \mathrm{kN} / \mathrm{m}^{2}$ for which the load incremental ratio is smallest at 0.25 , obtaining $m_{v}=6.2 \times 10^{-4} 1 /\left(\mathrm{kN} / \mathrm{m}^{2}\right), c_{v} *\left(=c_{v}\right)=0.02 \mathrm{~cm}^{2} / \mathrm{min}$, and $\alpha=0.005$, and applying this constant to other test results to reproduce the calculations of the tests. By changing the assumed value of the ratio of the primary consolidation $m_{p} / m_{v}$ as shown in the figure, calculation results close to the measurement value can be obtained.

In the calculation shown in Figure 5, the assumed value of $m_{p} / m_{v}$ that matches the test result of the load increment ratio $\Delta p=39.2 \mathrm{kN} / \mathrm{m}^{2}(\Delta p / p=0.25)$ is 0.5 . Figure 6 shows the results of the calculation and comparison by revising the value of $m_{p} / m_{v}$ to 0.7 and 0.9 . In the case of $m_{p} / m_{v}=0.9$ where the proportion of the primary consolidation increases, the shape of the calculated consolidation-time curve is significantly different from the measured values. The difference in the calculation result in Figure 6 indicates the rationality of the assumed $m_{p} / m_{v}$ value $(=0.5)$. It is considered that the load increment ratio affects the ratio of the primary consolidation and the secondary consolidation of the total consolidation.

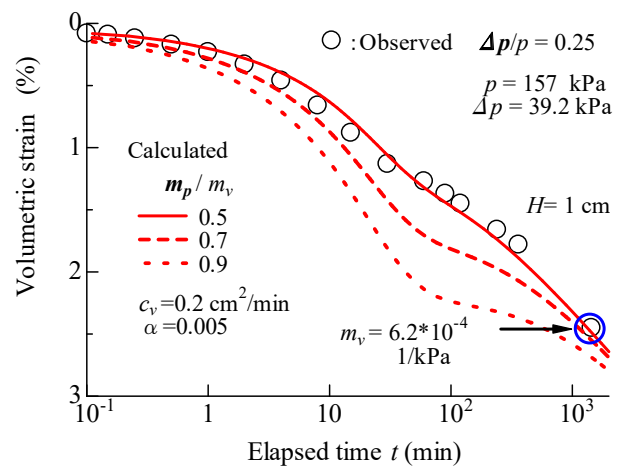

Figure 6. Settlement curves with differing primary consolidation ratios

\section{Conclution}

As a result of performing a consolidation test in which the load increment ratio was changed variously, test results different from the well-known past research results were obtained. In order to evaluate this result, it is necessary to examine whether physical properties etc. are indicators. The consolidation-time curve showing different shapes depending on the load increment ratio can be reproduced by changing the primary consolidation ratio. It is also considered that the smaller the load increment ratio, the smaller the proportion of primary consolidation.

\section{References}

1. G.A. Leonards, B.K. Ramiah, Time effects in the consolidation of clays, Am.Soc. Test., Matter. STP. 254, (1959).

2. C.B. Crawford, Interpretation of the consolidation test, Proc. ASCE, 90, SM5, (1964).

3. L. Bjerrum, Engineering geology of Norwegian normally consolidated marine clays as related to settlements of buildings, Geotechnique, 17(2), pp. 81-118, (1967).

4. T. Tsuchida et al., Comparison of results of consolidation testing using volumetric strain rate as the index, Thirty-fourth Japan National Conference on Geotechnical Engineering, pp. 487-488, (1999).

5. T. Mogami, Soil mechanics, Gihodo, pp.331-478, (1970).

6. T. Takeda, M. Sugiyama, M. Akaishi, H.W. Chang, Secondary compression behavior in one-dimensional consolidation test, Journal of GeoEngineering, TGS, 7(2), pp. 53-58, (2012). 